\title{
METODOLOGÍA PARA LA MEJORA ARQUITECTÓNICA DE REPOSITORIOS UNIVERSITARIOS
}

\author{
Adrià Fernández-Luna* \\ Xhotels \\ Mario Pérez-Montoro Gutiérrez ${ }^{* *}$ \\ Facultad de Biblioteconomía y Documentación. Universidad de Barcelona. \\ Javier Guallar ${ }^{* * *}$ \\ Facultad de Biblioteconomía y Documentación. Universidad de Barcelona.
}

\begin{abstract}
Resumen: Se propone la utilización de metodologías propias de la disciplina de la arquitectura de la información para mejorar el acceso a los contenidos de los repositorios universitarios o institucionales. Se ha observado que en la mayoría $(80 \%)$ de los repositorios universitarios se utiliza una implementación por defecto del software DSpace. El hecho de no adecuar la arquitectura del repositorio a las necesidades de la universidad y sus usuarios puede generar una mala experiencia de usuario llegando a generar búsquedas insatisfactorias en una de cada tres interacciones. Este obstáculo en la recuperación de documentos puede evolucionar en una falta de visibilidad de la producción científica de la institución. Por ello se hace una propuesta metodológica para mejorar la arquitectura, principalmente los sistemas de organización, de los repositorios basada en las técnicas de user persona y de card sorting empleando herramientas gratuitas como Google Forms, y se aplica la metodología a un estudio de caso: el Depósito Digital de la UB.

Palabras clave: Arquitectura de la información; experiencia de usuario; repositorios institucionales; producción científica; card sorting; user persona; metodología; estudio de caso.
\end{abstract}

Title: METHODOLOGY FOR THE ARCHITECTURAL IMPROVEMENT OF UNIVERSITY REPOSITORIES. Abstract: The article proposes the use of methodologies of the discipline of Information Architecture to improve access to the contents of institutional repositories. It has been observed that in the majority $(80 \%)$ of the university repositories a default implementation of the DSpace software is used. The fact of not adapting the architecture of the repository to the needs of the university and its users can generate a bad user experience, generating unsatisfactory searches in one of every three interactions. This obstacle in the recovery of documents can evolve in a lack of visibility of the scientific production of the institution. Therefore, a methodological proposal is made to improve the architecture (organization system, mainly) of the repositories based on the techniques of user persona and card sorting using free tools such as Google Forms, and this methodology is applied to a case study: Depósito Digital of the UB.

Keywords: Information architecture; user experience; institutional repositories; scientific production; card sorting; user persona; methodology; case study.

Copyright: (C) 2019 Servicio de Publicaciones de la Universidad de Murcia (Spain). Este es un artículo de acceso abierto distribuido bajo los términos de la licencia Creative Commons Reconocimiento 4.0 Internacional (CC BY 4.0).

\section{INTRODUCCIÓN}

Los repositorios o depósitos digitales son para las universidades una de las herramientas principales en la difusión de su producción científica, que es uno de los ejes centrales de la labor de estas instituciones (Expósito-García; Velasco-Morente, 2018). Según un estudio de Wiley Online Library (Pinfield et al., 2014) el modelo de difusión de estas instituciones evoluciona hacia los repositorios abiertos y el número de repositorios de libre acceso crece cada año a nivel mundial. Los repositorios, son por tanto cada vez más la herramienta principal de difusión de la producción científica de la universidad, que, al garantizar el libre acceso a la misma, consigue una mayor visibilidad y un notable impacto positivo en los índices de citaciones y en el posicionamiento en los rankings globales.

Debido a ese papel central en la difusión de la producción científica de las universidades, los repositorios han sido objeto de estudio por la literatura especializada en la última década, destacando los trabajos sobre la evaluación de la

\footnotetext{
*adriafluna@gmail.com

** perez-montoro@ub.edu

*** jguallar@gmail.com
}

Recibido: 01-01-2019; 2 ${ }^{\mathrm{a}}$ versión: 05-02-2019; aceptado: 28-02-2019.

FERNÁNDEZ LUNA, A.; PÉREZ-MONTORO GUTIÉRREZ, M. y GUALLAR DELGADO, J. Metodología para la mejora arquitectónica de repositorios universitarios. Anales de Documentación, 2019, vol. 22, $\mathrm{n}^{\circ} 2$. Disponible en: http://dx.doi.org/10.6018/analesdoc.356431. 
calidad de los mismos (Kim; Kim, 2008; Cassella, 2010; Barrueco Cruz et al., 2010; Primary Research Group, 2012; Barrueco Cruz et al., 2014; Serrano-Vicente; Melero; Abadal, 2014; Luca; Narayan, 2016; Serrano-Vicente; Melero; Abadal, 2018). Los enfoques de los estudios abarcan el marketing o promoción de los repositorios, su financiación, la cooperación con otros repositorios, las políticas de las instituciones al respecto o los servicios que ofrecen.

La revisión de la literatura permite constatar que el objetivo principal de los repositorios universitarios es el de dar a conocer los resultados de las propias investigaciones de la institución, así como garantizar su conservación. Sin embargo, el hecho de poner esa producción académica a libre disposición de cualquier usuario no basta para garantizar su acceso a ella. Los gestores de repositorios se han preocupado especialmente en procurar que los miembros de cada centro depositen sus trabajos en los mismos, confiando generalmente en programas recolectores o en buscadores como Google Scholar, como las herramientas que los usuarios externos a la institución utilizarán preferentemente para acceder a sus contenidos.

Sin embargo, más allá de confiar en la ayuda externa de un buscador, una mala arquitectura de cualquier sitio web (y un repositorio no deja de serlo), puede dificultar e incluso llegar a impedir el acceso a gran parte de la colección (Rosenfeld; Arango, 2015; Burford, 2011).

La arquitectura de la información (en adelante $\mathrm{AI}$ ) es la disciplina clave en el proceso de recuperación de la información cuyo objetivo es garantizar la satisfacción del usuario y reducir los costes derivados de la búsqueda en tiempo y recursos (Pérez-Montoro, 2010; Pérez-Montoro; Codina, 2016). Algunos estudios van más allá y afirman que una mala AI puede llegar a impedir que el usuario complete el proceso de recuperación (Nielsen, 2009), en este caso impidiendo que los usuarios lleguen a la documentación que están buscando en el repositorio. Se deduce de ello, que además de generar una mala experiencia de usuario, una mala AI puede suponer una reducción de visibilidad y a la larga un claro impacto negativo en los índices de citaciones y en los rankings de universidades.

La inversión en la creación y mantenimiento de los repositorios varía en cada institución. Según Serrano-Vicente, Melero y Abadal (2018), a nivel nacional el 66\% de las universidades españolas tienen personal a tiempo completo trabajando en sus repositorios, un porcentaje que es considerado insuficiente. En este contexto, garantizar una adecuada visibilidad y difusión de los documentos que contiene ayudaría a generar un retorno de la inversión positivo. Por todo ello, este trabajo estudiará los repositorios académicos desde un punto de vista muy poco tratado en la literatura, como es el de su arquitectura de información.

\section{OBJETIVOS Y METODOLOGÍA}

La finalidad de este trabajo es ofrecer una propuesta metodológica centrada en los usuarios que permita el diseño adecuado de la estructura arquitectónica, principalmente los sistemas de organización, de los repositorios universitarios. Para cubrir esa finalidad hemos planteado una serie de objetivos.

- O1. En primer lugar, realizar un análisis de los repositorios y de la tecnología asociada a los mismos de una muestra significativa de los mismos en el contexto español.

- O2. En segundo lugar, y es el objetivo fundamental o central de este trabajo, proponer una estrategia metodológica estandarizada para diseñar la estructura arquitectónica, principalmente los sistemas de organización, de un repositorio institucional universitario.

- O3. Por último, contrastar el alcance y consistencia de la propuesta metodológica anterior, implementándola en un caso concreto.

La metodología de investigación empleada, siempre en relación con los objetivos anteriores, es la siguiente, utilizando las denominaciones recogidas en Guallar et al. (2017):

- Para cubrir el O1, se realiza una investigación descriptiva basada en la técnica de análisis de contenido sobre una muestra formada por los repositorios de las 15 universidades españolas más importantes utilizando como criterio de selección el de la producción científica.

- Para el O2, la propuesta metodológica se puede inscribir en el método de investigación de diseño de sistemas de información, y se articula sobre el desarrollo de dos técnicas de investigación básicas en diseño de interacción: las técnicas de user persona y de card sorting, que se explicarán con más detalle en los apartados 3.2.1 y 3.2.2.

- Para cubrir el objetivo O3, se emplea la técnica de investigación del estudio de caso, y se aplica la propuesta metodológica al rediseño arquitectónico, principalmente los sistemas de organización, del Depósito Digital, el repositorio institucional de la Universidad de Barcelona (en adelante, UB).

El período de recogida de datos del estudio fue entre abril y mayo de 2018. 


\section{RESULTADOS}

Fruto de la aplicación de la metodología anteriormente descrita se obtuvieron los siguientes resultados, que se muestran siguiendo la estructura de los tres objetivos expuestos antes:

- Se introduce en primer lugar el análisis de los repositorios y la tecnología asociada a los mismos de las 15 universidades españolas más importantes respecto al criterio de la producción científica.

- Se presenta una estrategia metodológica estandarizada para diseñar la estructura arquitectónica, principalmente los sistemas de organización, de un repositorio institucional universitario.

- Se aplica la propuesta al Depósito Digital de la UB.

\subsection{Repositorios y tecnología}

Con la finalidad de obtener una muestra de repositorios españoles relevantes, se han articulado los datos de las 10 universidades españolas más importantes según el Scimago Institutions Rankings (2017) y las 10 universidades más importantes de España según el ranking ISI, recogidas y analizadas, por ejemplo, en el trabajo de Torres-Salinas et al. (2011). De este cruce de datos, omitiendo la duplicidad de resultados, se identificaron las 15 universidades mejor valoradas de España de acuerdo a su producción científica.

A continuación, se analizaron sus repositorios y las tecnologías empleadas en ellos. Los datos se muestran en la tabla I y el gráfico 1, agrupados por el software utilizado y ordenados por su versión de instalación.

\begin{tabular}{|c|c|c|c|c|c|}
\hline Universidad & $U R L$ & $N^{o}$ Documentos & Software & Versión & $\boldsymbol{U} \boldsymbol{I}$ \\
\hline Sevilla & https://idus.us.es & 55.130 & DSpace & 6.1 & Custom \\
\hline Politécnica de Valencia & https://riunet.upv.es & 74.173 & DSpace & 5.6 & Custom \\
\hline País Vasco & https://addi.ehu.es & 9.644 & DSpace & 5.6 & Mirage2 \\
\hline Santiago Compostela & https://minerva.usc.es & 16.060 & DSpace & 5.6 & Mirage2 \\
\hline Barcelona & http://diposit.ub.edu & 33.819 & DSpace & 5.5 & Mirage2 \\
\hline Pompeu Fabra & https://repositori.upf.edu & 14.837 & DSpace & 5.5 & Custom \\
\hline Navarra & https://dadun.unav.edu & 40.995 & DSpace & 5.4 & Custom \\
\hline Carlos III & https://e-archivo.uc3m.es & 23.889 & DSpace & 5.4 & Custom \\
\hline Politécnica Cataluña & https://upcommons.upc.edu & 105.457 & DSpace & 4.2 & Custom \\
\hline Autónoma Madrid & https://repositorio.uam.es & 33.340 & DSpace & 3.2 & XMLUI \\
\hline Valencia & http://roderic.uv.es & 45.283 & DSpace & (2015) $[4-5]$ & XMLUI \\
\hline Granada & http://digibug.ugr.es & 39.431 & DSpace & (2008) [1.5] & XMLUI \\
\hline Complutense & http://eprints.ucm.es & 37.640 & E-Prints & 3.3.12 & - \\
\hline Zaragoza & https://zaguan.unizar.es & 41.996 & Invenio & 1.2 & - \\
\hline Autónoma Barcelona & https://ddd.uab.cat & 167.378 & Invenio & 1.1.6 & - \\
\hline
\end{tabular}

Tabla I. Comparativa de tecnologías utilizadas en los repositorios universitarios en 2018. 


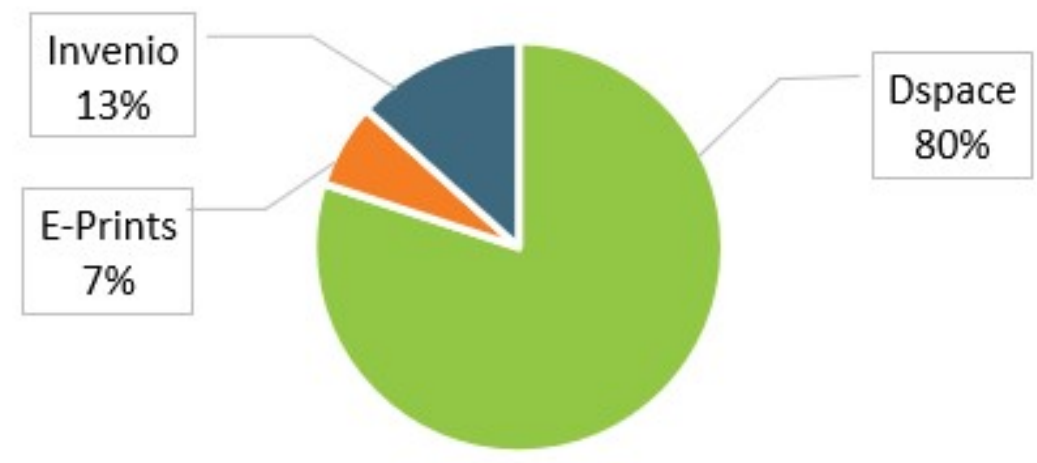

Figura 1. Grafico de uso de softwares para repositorios en las universidades españolas.

Se pudo apreciar cómo, en la muestra de 15 repositorios españoles, el 80\% de ellos utilizaban el software DSpace, el 13\% Invenio y sólo el 7\% E-Prints. Estos resultados coinciden con los mostrados en otros trabajos, donde, con una muestra más representativa de 46 repositorios españoles, se señala que hasta en un $87 \%$ de casos abordados se utiliza DSpace para su gestión (Serrano-Vicente; Melero; Abadal, 2018).

De los 12 repositorios analizados creados con DSpace, se pudo comprobar que el 50\% estaban aplicando la implementación por defecto utilizando la interfaz de Mirage2, y aunque el resto añadían funcionalidades y diseños propios de la institución, la arquitectura era común en todos ellos.

Se constató por tanto que se trata de una propuesta arquitectónica pobre y poco articulada, derivada directamente de la propuesta tecnológica, y no testeada a partir de estudios de usuarios. En esos sistemas desarrollados por defecto no existe un sistema de navegación temático, que permita navegar y acceder a los documentos administrados. En el mejor de los casos, solo hay una caja de búsqueda complementada por un sistema de navegación (de un solo nivel) para acceder a los tipos formales de documentos. Como la literatura especializada ha señalado, las estructuras por defecto, que no se ajustan a las necesidades concretas de los usuarios o las instituciones, tienden a generar malas experiencias de usuario debido a la diversidad en las técnicas y herramientas de recuperación de que dispone la institución (Bates, 1989).

\subsection{Metodología para el rediseño arquitectónico}

Las limitaciones de esta práctica común justifican la necesidad de nuevas propuestas de arquitectura de la información de los repositorios institucionales. La propuesta metodológica sugerida, que puede implementarse sin la utilización de software propietario de pago, se estructura en dos partes.

Por un lado, en primer lugar, se realizaría un análisis de usuarios utilizando el método de user persona. Con este se persigue analizar y evaluar los distintos perfiles de usuarios que utilizan el repositorio haciendo inciso en cuáles son sus necesidades concretas de información, y cuáles son sus habilidades de recuperación.

Y, por otro, en segundo lugar, se aplicaría una variante de la técnica del card sorting que permitiría extraer de los usuarios la propia estructura de organización y navegación del entorno digital de acorde a su modelo mental e implementarlo o usarlo como referencia para comparar y analizar la estructura del repositorio.

\subsubsection{Método user persona}

El denominado método del user persona consiste en la definición de personajes creada a partir del análisis de los usuarios, y sirve de ejemplo para el estudio de casos durante el desarrollo de la user experience $(U X)$ o experiencia de usuario (Garret, 2011). O, dicho de otra manera, consiste en la identificación de los usuarios arquetípicos del sistema, su target o público objetivo, para, poniéndonos en su piel, poder resolver propuestas de diseño sin tener que consultar a usuarios reales.

Para realizarlo, se identifican en primer lugar, los principales perfiles de usuarios. En el caso de un repositorio universitario se sugieren los siguientes arquetipos:

- Personal docente e investigador

- Personal de administración y servicios 
- Alumnado

- Usuarios externos

En segundo lugar, para cada perfil de usuario hay que definir un conjunto de datos que nos permitan entender cuál será su comportamiento, como por ejemplo el grado de representación, su dominio de habilidades informacionales, sus necesidades y las metodologías que despliegan.

- Representación: estimación entre el total de usuarios del repositorio y los que pertenecen a este perfil concreto.

- Dominio: clasificación de los usuarios según sus habilidades informacionales o de recuperación de información (Russell-Rose, 2013). Estos pueden ser:

- Novatos en el campo de estudio y en las técnicas de búsqueda (Double Novice).

- Novatos en el campo de estudio y expertos en las técnicas de búsqueda (Novice-Expert).

- Expertos en el campo de estudio y novatos en las técnicas de búsqueda (Expert-Novice).

- Expertos en el campo de estudio y en las técnicas de búsqueda (Double Expert).

- Necesidades concretas de información: se debe dar respuesta a la pregunta: ¿Para qué utilizará el usuario el repositorio?

- Métodos de búsqueda preferidos: identificar cuáles son, de los métodos y herramientas de interacción, las que utiliza más el usuario. Estas pueden ser el sistema de búsqueda, la navegación, búsqueda exploratoria o la búsqueda por identificador, entre otros. Si se dispone de más de un sistema de navegación es prudente obtener datos sobre cuál de ellos es más utilizado.

\subsubsection{Técnica del card sorting}

Con los resultados obtenidos en la creación de los user persona se puede iniciar el diseño del card sorting. Se trata de una técnica que persigue diseñar sistemas de clasificación (normalmente sistemas de navegación para sistemas o páginas web) que se correspondan con las expectativas organizacionales de los usuarios que van a utilizar ese sistema de información. En un sentido práctico, este método consiste en facilitar a los usuarios el conjunto de tarjetas que se quieren categorizar y pedirles que las agrupen según su propio criterio. Esto permite reflejar el modelo mental de los usuarios y predecir cómo esperan encontrar organizada la información en un sitio web.

Existen dos modelos de card sorting: el cerrado y el abierto. Cada uno de ellos se implementa dependiendo de los intereses de diseño que se persigan.

En el card sorting cerrado las categorías en las que los usuarios han de organizar las etiquetas vienen predefinidas por el investigador que ha diseñado el test. Esto da menos libertad a los usuarios e impone un modelo cognitivo concreto al que los usuarios se deben adaptar. Sin embargo, es apropiado cuando lo que se pretende es analizar la adecuación de una categorización ya existente.

En el card sorting abierto las categorías no están previamente definidas. En lugar de ello se da libertad a los usuarios para que agrupen las etiquetas bajo un criterio menos restrictivo y luego den un nombre a sus agrupaciones. Este tipo está especialmente indicado si en lugar de evaluar un sistema de organización existente lo que se persigue es crear uno nuevo.

En ambos casos, en primer lugar hay que seleccionar la muestra de usuarios. Para ello se propone utilizar el estudio de Tullis (2004) cuyos principales resultados se han reflejado en la tabla II, y seleccionar la muestra acorde a las necesidades y recursos de la institución, valorando el número de usuarios al que se tiene acceso, el coste del análisis y el porcentaje de coincidencia que se persigue. Este indica con cuanta frecuencia coincidirán las respuestas de los usuarios.

\begin{tabular}{|c|c|c|c|}
\hline Usuarios & Coincidencia & Usuarios & Puntos porcentuales \\
\hline 5 & $75 \%$ & - & - \\
\hline 15 & $90 \%$ & 10 & 0,15 \\
\hline 30 & $95 \%$ & 15 & 0,05 \\
\hline 60 & $98 \%$ & 30 & 0,03 \\
\hline
\end{tabular}

Tabla II. Relación de $\mathbf{n}^{0}$ de usuarios y ratio de coincidencia en las respuestas. Fuente: Tullis (2004).

Para lograr una mayor representatividad, la muestra debe ser aleatoria estratificada (Corbetta, 2007). Cada perfil de usuario constituye un estrato de la muestra y su representatividad será relativa al número total de usuarios del repositorio. 
Descrita la composición de la muestra, pasamos ahora a implementar el card sorting. Para desarrollar esta técnica, existen distintas herramientas. En la tabla III, se muestra una selección de las opciones disponibles analizadas.

\begin{tabular}{l|l|l} 
Herramienta & Gratuidad & Distribuible en línea \\
\hline Optimal Sort & Máximo 10 participantes & Sí \\
\hline Proven by Users & Máximo 3 participantes & Sí \\
\hline Usability Test & Prueba de 48h & Sí \\
\hline Simple card sorting & No & Sí \\
\hline UserZoom & No & Sí \\
\hline SynCaps & No & Sí \\
\hline UXsort & Sí & No \\
\hline CardZort & Sí & No \\
\hline xSort & Sí & No \\
\hline AutocardSorter & Sí & No \\
\hline \multicolumn{2}{l}{ Tabla III. Comparativa de herramientas para card sorting (2018). Fuente: Elaboración propia. }
\end{tabular}

Como se puede apreciar, ninguna de las herramientas disponibles permite realizar un card sorting de forma gratuita, online y para una muestra de 30 usuarios, que es el número mínimo generalmente aconsejado en la bibliografía especializada (Spencer, 2009). Existe una alternativa para hacerlo sin tener que utilizar un software propietario de pago. Concretamente, utilizando una herramienta de formularios gratuita online. De las actualmente disponibles se han testeado Google Forms, Microsoft Forms, Trello y Disroot Polls.

De estas, se han descartado la mayoría por los siguientes motivos. Por un lado, Microsoft Forms y Disroot tienen un máximo de opciones en las preguntas de tipo "matriz de selección" (el usuario tiene que asignar un valor a las celdas de cada fila de acuerdo al título de la columna), lo que presenta limitaciones clave a la hora de dar opciones de categorización al usuario. Por otro lado, Trello, a pesar de ser una herramienta muy parecida a un card sorting real no permite dar instrucciones precisas a los usuarios y además, hay que generar un enlace y tableros únicos para cada usuario de los que se deberán extraer en formato JSON añadiendo pasos adicionales para lograr tratar los datos debidamente.

Habiendo descartado las principales alternativas, se propone trabajar con Google Forms utilizando las preguntas de la opción de "matriz de selección". En este caso, los usuarios deben decidir bajo qué grupo incluirían cada una de las etiquetas.

Para valorar la adecuación de la herramienta, durante el proceso de diseño de la metodología se fueron realizando pequeños estudios de usuarios, cuyo objetivo fue analizar el comportamiento y dificultades que estos presentaban durante la realización del card sorting con Google Forms.

En el estudio final, con una muestra de 5 usuarios, ninguno de ellos tuvo dificultades para entender cuál era el objetivo de la tarea y cómo debía llevarse a cabo con la única información de los textos de ayuda que complementaban la matriz de selección y sin ningún tipo de asistencia externa. De este modo, se respalda la propuesta de enviar telemáticamente el test sin necesidad de dar soporte directo a los usuarios ni estar junto a ellos a lo largo de todo el proceso (Nielsen, 2012).

Para un card sorting doble de 30 y 20 etiquetas los usuarios no tuvieron que invertir más de 10 minutos en total para completarlo.

\begin{tabular}{|l|l|l|l|} 
Usuario & Tiempo M. (30 etiquetas) & Tiempo C. (20 etiquetas) & T. Total \\
\hline R. & $05: 40$ & $04: 10$ & $09: 50$ \\
\hline B. & $04: 00$ & $02: 52$ & $06: 52$ \\
\hline A. & $05: 58$ & $03: 01$ & $08: 59$ \\
\hline J. & $05: 53$ & $03: 53$ & $09: 46$ \\
\hline E. & $03: 03$ & $01: 48$ & $04: 51$ \\
\hline X & $\mathbf{0 4 : 5 5}$ & $\mathbf{0 3 : 0 9}$ & $\mathbf{0 8 : 0 4}$ \\
\hline
\end{tabular}

Tabla IV. Tiempos card sorting utilizando un formulario de Google. Fuente: Elaboración propia. 
Gracias a esta información se pudo inferir que el tiempo total del test parecía estar dentro de unos límites aceptables para que los usuarios no sintieran fatiga u optaran por abandonar el card sorting antes de terminar.

En el primer test de usuarios, se descartó la opción de crear categorías abiertas utilizando distintos tipos de pregunta. La propuesta consistía en utilizar la matriz de selección dejando categorías sin valor semántico como (Categoría 1, u Opción A) y en una pregunta adicional de "texto libre" permitir al usuario dar valor semántico a esas categorías definiendo qué etiquetas usarían. Se comprobó que estas preguntas sí generaban confusión en los usuarios, los cuales optaban por preguntar al observador cómo debían responder o las dejaban en blanco. Este suceso generaba un riesgo en la obtención de datos y suponía una incompatibilidad con enviar el test de forma telemática. Por ese motivo se propone utilizar Google Forms solo para llevar a cabo card sorting de tipo cerrado.

En caso de necesitar realizar un card sorting de tipo abierto con una muestra grande de usuarios, en la propuesta metodológica se sugiere un paso previo que permita obtener las etiquetas propuestas por los usuarios y que serán utilizadas en la "matriz de selección".

A continuación, se detallan los pasos necesarios para llevar a cabo la propuesta metodológica.

\section{a) Paso previo}

Para poder recoger el modelo mental de los usuarios en el card sorting cerrado, se propone implementar esta técnica en dos fases. Para ello, se necesita una muestra de 60 usuarios. 30 usuarios realizarán un card sorting abierto empleando una herramienta dedicada, mientras que los otros 30 realizarán el cerrado con Google Forms. El objetivo es poder cruzar los datos del card sorting cerrado, llevado a cabo con una muestra de 30 usuarios, con el fin de obtener una mayor representatividad de los resultados. El principal inconveniente de este proceso es que añade una capa de complejidad e inversión de tiempo y recursos que puede no estar al alcance de todas las organizaciones.

El primer paso, es crear 3 card sorting abiertos en la herramienta Optimal Sort, para ello se deben haber extraído con anterioridad las etiquetas de segundo nivel del sistema de organización, dejando las de primer nivel como categorías abiertas en la herramienta.

En el segundo paso, hay que dividir la muestra en 3 grupos de 10 usuarios. A cada uno de ellos se le facilitará un enlace de Optimal Sort. La herramienta no permitirá cruzar los datos en bruto, pero sí podremos obtener las etiquetas sugeridas de primer nivel. Estas serán las utilizadas para realizar el card sorting cerrado.

\section{b) Card sorting cerrado}

El objetivo es crear un card sorting cerrado utilizando la herramienta de Google Forms. A la hora de crear las preguntas, existe una pregunta de tipo "matriz de selección", en ella se puede definir cada fila de la matriz como una página o contenido a categorizar (Figura 2). Mientras que en cada columna de la matriz se deben introducir las categorías. Los nombres o etiquetas de las categorías pueden ser los obtenidos en el "paso previo" o, si se ha omitido, se deben utilizar las que existen actualmente en el repositorio.

De este modo, los usuarios obtendrán una matriz de selección en la que en las columnas verán categorías y en las filas las etiquetas que deben categorizar. La matriz solo permite una respuesta por fila de modo que permite prevenir errores de los usuarios.

\section{Categoría 1 Categoría 2 Categoría 3 Categoría n}

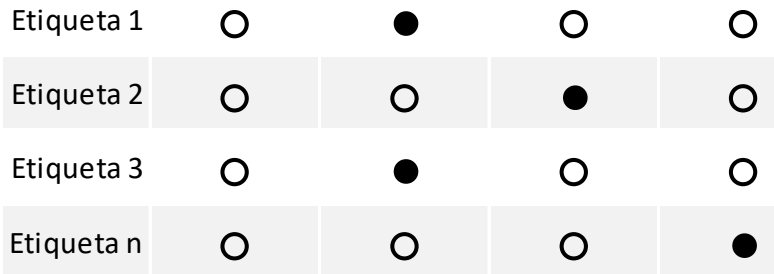

Figura 2. Ejemplo de matriz de selección para un card sorting. Fuente: Elaboración propia.

Google Forms generará una tabla de resultados en la que cada fila será una respuesta de usuario, mientras que cada columna será una de las tarjetas que se le han dado al usuario para que categorice. 


\begin{tabular}{l|l|l|l} 
Marca temporal & Tarjeta 1 & Tarjeta 2 & Tarjeta n \\
\hline T. Respuesta usuario 1 & Categoría 1 & Categoría 2 & Categoría $\mathrm{n}$ \\
\hline T. Respuestas usuario 2 & Categoría 1 & Categoría 3 & Categoría $\mathrm{n}$ \\
\hline T. Respuestas usuario n & Categoría n & Categoría $\mathrm{n}$ & Categoría $\mathrm{n}$ \\
\hline \multicolumn{1}{l}{ Tabla V. Ejemplo de resultados del card sorting } & en Google Forms. Fuente: Elaboración propia
\end{tabular}

Estos datos se deben exportar a una hoja de cálculo para procesarlos automáticamente a partir de una matriz de popularidad.

\section{c) Matriz de popularidad}

La matriz de popularidad mide la frecuencia con la que una tarjeta aparece en cada categoría, y nos permite inferir cual es la categoría más popular para cada una de las tarjetas.

\begin{tabular}{|l|c|c|c|} 
Tarjeta & Categoría 1 & Categoría 2 & Categoría n \\
\hline Tarjeta 1 & 7 & 1 & $\mathrm{n}$ \\
\hline Tarjeta 2 & 5 & 3 & $\mathrm{n}$ \\
\hline Tarjeta $\mathrm{n}$ & $\mathrm{n}$ & $\mathrm{n}$ & $\mathrm{n}$ \\
\hline
\end{tabular}

Tabla VI. Ejemplo de resultados del card sorting en Google Forms. Fuente: Elaboración propia.

Estos valores se pueden obtener automáticamente si utilizamos una hoja de cálculo y la función "CONTAR.SI". La función debe hacer referencia a la tabla principal donde tenemos los datos del card sorting. Por ejemplo: =CONTAR.SI(“Columna Tarjeta 1 en tabla de datos"; "Categoría 1")

\begin{tabular}{l|l} 
Software & Expresión \\
\hline MS Excel / LibreOffice Español & $=$ CONTAR.SI(rango;criterio) \\
\hline MS Excel / LibreOffice Internacional & $=$ COUNTIF(rango,criterio) \\
\hline Google Sheets & $=$ COUNTIF(rango,criterio) \\
\hline
\end{tabular}

Tabla VII. Resumen de funciones. Fuente: Elaboración propia.

A partir de estos datos se puede, además, obtener el índice de concurrencia. Calculado a partir del número total de categorías empleadas en cada tarjeta ( $1 / \mathrm{n}^{\mathrm{o}}$ categorías). Un índice de concurrencia bajo indica que los usuarios no tienen un único criterio para categorizar las tarjetas, mientras que un índice de concurrencia alto indica una mayor uniformidad en el criterio.

\section{d) Dendrograma}

El dendrograma (o diagrama de árbol) permite ver las agrupaciones lógicas que han propuesto los usuarios por la proximidad en la que han categorizado las tarjetas. Una herramienta libre disponible para ello es Hierarchical Clustering de Wessa-Free Statistics Software (https://wessa.net/rwasp_hierarchicalclustering.wasp).

Para utilizarlo basta con copiar los valores obtenidos de la matriz de popularidad en el campo “data X”. Se pueden cambiar algunas opciones de personalización como el método de clusterizado, la orientación del dendrograma o el formato resultante.

Con el método de clusterizado ward se consigue minimizar el número de nodos haciendo más fácil la interpretación del dendrograma y facilitando la toma de decisiones a la hora de añadir, eliminar o seleccionar categorías. 


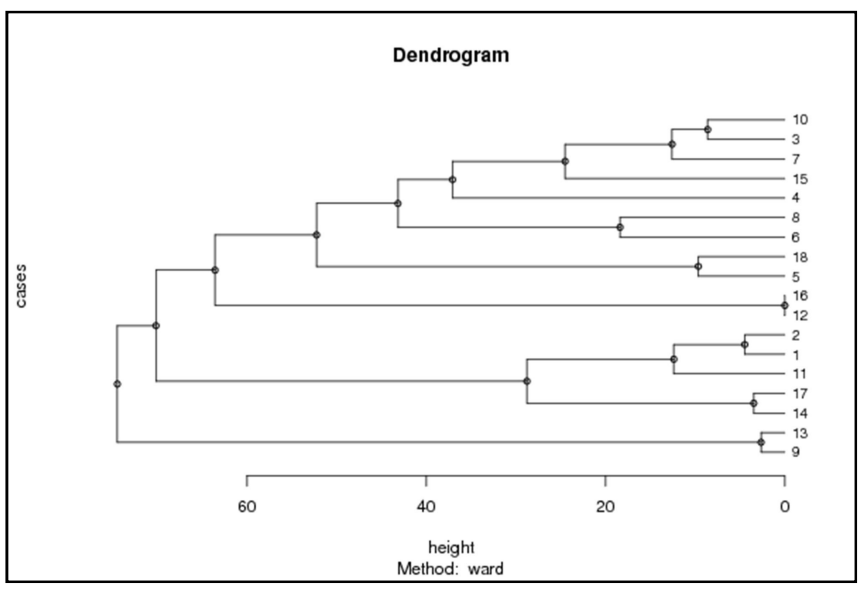

Figura 3. Ejemplo de dendrograma empleando el método ward, Fuente: Wessa-Free Statistics Software.

Para realizar la propuesta de Sistema de Organización basta con analizar las agrupaciones que confluyen en cada nodo y las categorías mejor valoradas en la matriz de popularidad. De este modo se puede ver en qué punto separar una agrupación de otra y crear una categoría nueva. Por ejemplo:

\begin{tabular}{|l|l|l|l|l|}
\hline Dendrograma & Tarjeta & Categoría más popular & Agrupación \\
\hline & Biología & Ciencias aplicadas & 3 \\
\hline & Ciencias médicas & Ciencias aplicadas & 3 \\
\hline & Arquitectura & Ciencias aplicadas & 3 \\
\hline & Astronomía & $\begin{array}{l}\text { Matemáticas y ciencias } \\
\text { naturales }\end{array}$ & 2 \\
\hline & Física & $\begin{array}{l}\text { Matemáticas y ciencias } \\
\text { naturales }\end{array}$ & 2 \\
\hline & Ciencias sociales & 1 \\
\hline
\end{tabular}

Tabla VIII. Ejemplo de agrupaciones cruzando datos de la matriz de popularidad y el dendrograma. Fuente: Elaboración propia. 
Flujo de trabajo de la propuesta metodológica:

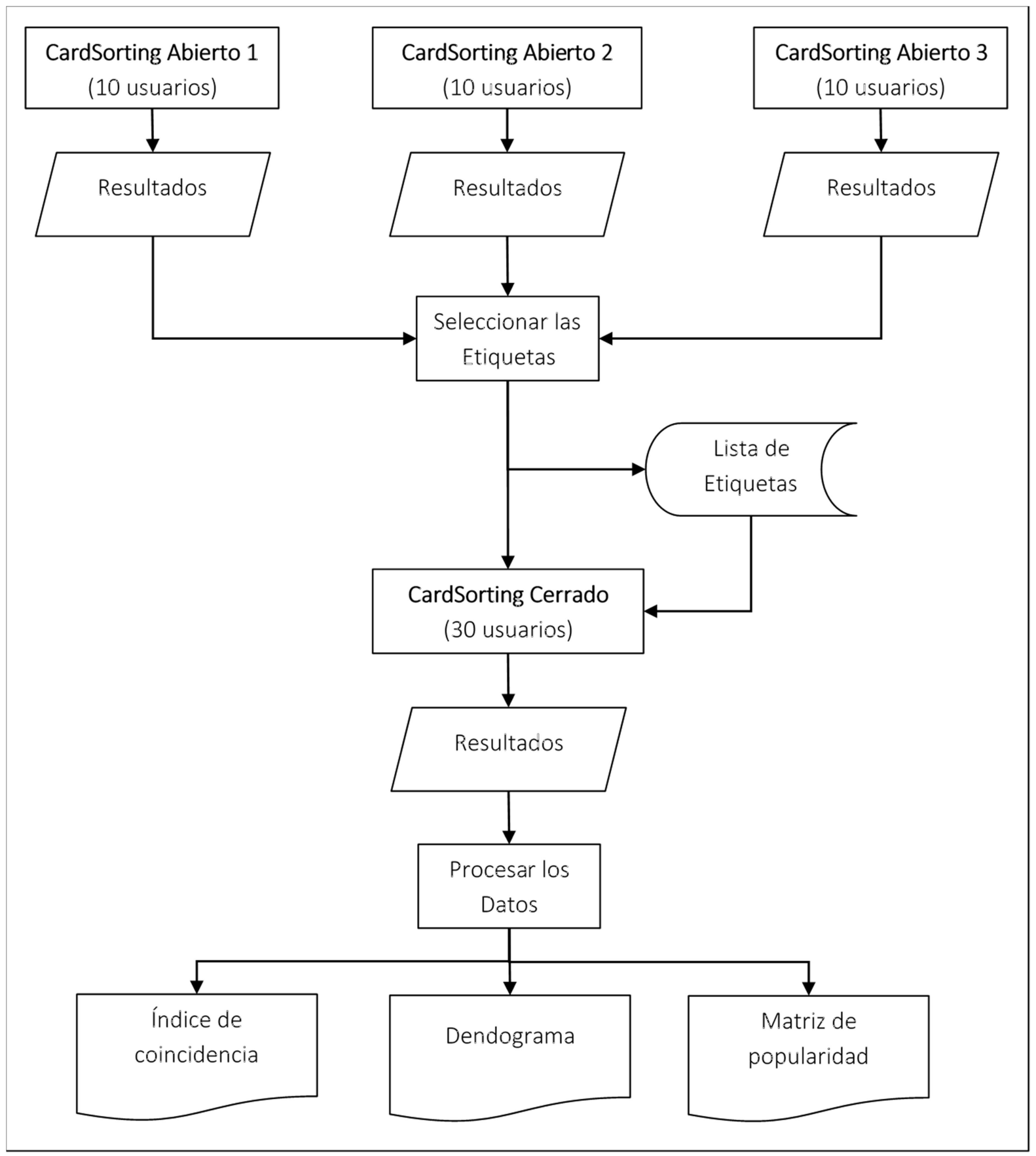

Figura 4. Resumen del flujo de trabajo. Fuente: Elaboración propia.

\section{ESTUDIO DE CASO}

Para probar el alcance y la consistencia de la propuesta metodológica se ha implementado en el análisis del Depósito Digital, el repositorio institucional de la UB. Con ello se puede evaluar la adecuación del repositorio a las necesidades de sus usuarios y al mismo tiempo las dificultades que puede presentar la propuesta metodológica elaborada previamente. 


\subsection{Creación de los user persona}

Siguiendo la propuesta metodológica, se han identificado 4 perfiles de usuarios distintos, PDI, alumno, PAS, y personal externo. En la tabla IX se muestra el resumen de los elementos clave necesarios que se han utilizado para crear los user persona.

\begin{tabular}{l|l|l|l|l|}
\multicolumn{2}{l}{ Nombre } & \multicolumn{1}{c}{ Tipo de usuario } & \multicolumn{1}{c}{$\begin{array}{l}\text { Método } \\
\text { búsqueda preferido }\end{array}$} & \multicolumn{1}{l}{ Necesidades de información } \\
\hline PDI & Angela Barranco & Double Expert & Por identificador & $\begin{array}{l}\text { Bibliografía básica de sus asignaturas } \\
\text { Actualización del material docente }\end{array}$ \\
\hline Alumno & Juan Díaz & Double Novice & Exploratoria & $\begin{array}{l}\text { Documentos de referencia para su } \\
\text { TFG. } \\
\text { Fuentes biográficas. }\end{array}$ \\
\hline Externo & Ruth Pales & Expert - Novice & Facetada & $\begin{array}{l}\text { Documentación institucional y planes } \\
\text { docentes. }\end{array}$ \\
\hline PAS & Alfredo Llácer & Novice-Expert & Exploratoria & $\begin{array}{l}\text { Memorias anuales y documentación } \\
\text { relativa a los KPI de la institución. }\end{array}$ \\
\cline { 2 - 5 }
\end{tabular}

Tabla IX. Resumen Users Persona. Fuente: Elaboración propia.

\subsection{Card sorting}

Dado que el objetivo es analizar el estado actual del sistema de organización del Depósito Digital, se ha omitido el paso previo detallado anteriormente y necesario para un card sorting abierto, y se ha realizado directamente un card sorting cerrado con Google Forms. De este modo, los usuarios emplearán las categorías del sistema de organización actual y será más fácil identificar cuáles son sus puntos fuertes y débiles a fin de determinar si la propuesta de arquitectura actual es adecuada o no.

En el estudio de caso se ha decidido trabajar con una muestra de 30 participantes. De acuerdo con el estudio de Tullis, esta muestra generará un índice de coincidencia en las respuestas del 95\%, y dará un margen de error muy amplio en caso de que algún participante no lograra completar las tareas asignadas. La representatividad de los estratos de la muestra viene determinada por los porcentajes de uso del Depósito Digital de cada uno de los perfiles, cuyo dato se ha obtenido de la Memoria Anual de la UB, y quedan reflejados en la tabla X.

\begin{tabular}{|l|c|r|r|}
\hline Estratos/Perfiles & \% de usuarios & Muestra & Muestra| \\
\hline PDI & $63 \%$ & 18,9 & 19 \\
\hline Alumno & $22 \%$ & 6,6 & 7 \\
\hline Externo & $11 \%$ & 3,3 & 3 \\
\hline PAS & $4 \%$ & 1,2 & 1 \\
\hline Total & 1 & 30 & 30 \\
\hline
\end{tabular}

Tabla X. Ejemplo de muestra aleatoria estratificada. Fuente: Elaboración propia.

\subsection{Resultados del card sorting}

Los datos obtenidos del card sorting se recogen en la tabla XI. En esta se ha cuantificado el número de tarjetas que los usuarios situaron en cada categoría.

\begin{tabular}{|c|c|c|c|c|c|c|c|}
\hline Tarjeta & $\begin{array}{l}\stackrel{\Xi}{\Xi} \\
\stackrel{\Xi}{\Xi} \\
z\end{array}$ & 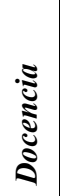 & 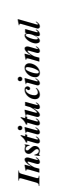 & 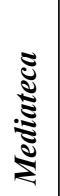 & हैं & 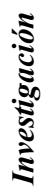 & : \\
\hline Datos Institucionales & 1 & 0 & 30 & 0 & 0 & 0 & 0 \\
\hline RIDOC (Recursos de Información para la Docencia) & 2 & 24 & 0 & 3 & 1 & 1 & 0 \\
\hline Memorias de los cursos académicos - Universidad de Barcelona & 3 & 4 & 22 & 2 & 1 & 0 & 1 \\
\hline TECNOLOGIA EDUCATIVA (TAC/TIC) & 4 & 11 & 0 & 1 & 16 & 1 & 0 \\
\hline Agencia de Políticas de Calidad & 5 & 2 & 24 & 1 & 0 & 1 & 0 \\
\hline
\end{tabular}




\begin{tabular}{|l|c|c|c|c|c|c|c|}
\hline INNOVADOC (Documentos de Innovación Docente) & 6 & 18 & 2 & 2 & 1 & 4 & 0 \\
\hline Datos de Investigación & 7 & 0 & 4 & 0 & 0 & 25 & 1 \\
\hline Centro de Recursos para el Aprendizaje e Investigación (CRAI-UB) & 8 & 6 & 10 & 6 & 1 & 6 & 1 \\
\hline Planes Docentes - Guías del estudiante & 9 & 22 & 6 & 0 & 2 & 0 & 0 \\
\hline Histórico UB (Anuarios, Memorias...) & 10 & 0 & 22 & 4 & 2 & 0 & 1 \\
\hline Imagen Corporativa y Márquetin UB & 11 & 0 & 26 & 3 & 1 & 0 & 0 \\
\hline Mediateca - Investigación & 12 & 0 & 1 & 18 & 0 & 11 & 0 \\
\hline Mediateca - Institucional & 13 & 1 & 5 & 23 & 0 & 1 & 0 \\
\hline Cursos de verano de la Universidad de Barcelona & 14 & 18 & 9 & 0 & 2 & 1 & 0 \\
\hline Software de Investigación & 15 & 1 & 1 & 1 & 19 & 8 & 0 \\
\hline Publicaciones de proyectos de investigación financiados por la UE & 16 & 0 & 1 & 5 & 0 & 24 & 0 \\
\hline Artículos publicados en revistas & 17 & 1 & 0 & 9 & 0 & 20 & 0 \\
\hline Libros / Capítulos de libros & 18 & 3 & 0 & 14 & 0 & 11 & 1 \\
\hline Comunicaciones a congresos & 19 & 1 & 5 & 4 & 2 & 18 & 0 \\
\hline Exposiciones & 20 & 2 & 13 & 8 & 2 & 2 & 0 \\
\hline Documentos de trabajo & 21 & 8 & 6 & 4 & 1 & 8 & 2 \\
\hline Divulgación y Prensa & 22 & 0 & 15 & 9 & 1 & 4 & 0 \\
\hline Software creado por el alumnado & 23 & 4 & 0 & 0 & 21 & 3 & 1 \\
\hline Centro de Estudios Internacionales de la UB & 24 & 7 & 19 & 0 & 0 & 4 & 0 \\
\hline Seminarios de Investigación & 25 & 4 & 1 & 1 & 0 & 22 & 1 \\
\hline Ciencia Histórica de la UB & 26 & 4 & 15 & 5 & 1 & 3 & 1 \\
\hline Tesis Doctorales - Departamento Ecología & 27 & 2 & 0 & 0 & 0 & 6 & 22 \\
\hline Tesis Doctorales - Departamento - Dibujo & 28 & 3 & 0 & 0 & 0 & 6 & 21 \\
\hline Tesis Doctorales - Escuela Universitaria de Enfermería & 29 & 2 & 0 & 0 & 0 & 6 & 22 \\
\hline OMADO (Objetos y Materiales Docentes) & 30 & 24 & 0 & 5 & 0 & 0 & 0 \\
\hline
\end{tabular}

Tabla XI. Resultados obtenidos. Fuente: Elaboración propia.

A partir de los datos obtenidos, se ha generado la matriz de popularidad, tabla XII. En ella queda reflejada la distribución estadística de las tarjetas para cada una de las categorías.

\begin{tabular}{|c|c|c|c|c|c|c|c|}
\hline Tarjeta & 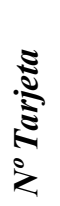 & 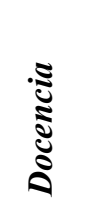 & 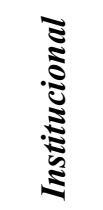 & 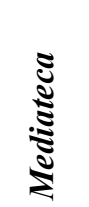 & है & 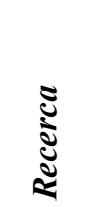 & 离 \\
\hline Datos Institucionales & 1 & $0 \%$ & $100 \%$ & $0 \%$ & $0 \%$ & $0 \%$ & $0 \%$ \\
\hline RIDOC (Recursos de Información para la Docencia) & 2 & $83 \%$ & $0 \%$ & $10 \%$ & $3 \%$ & $3 \%$ & $0 \%$ \\
\hline Memorias de los cursos académicos - Universidad de Barcelona & 3 & $13 \%$ & $73 \%$ & $7 \%$ & $3 \%$ & $0 \%$ & $3 \%$ \\
\hline TECNOLOGIA EDUCATIVA (TAC/TIC) & 4 & $38 \%$ & $0 \%$ & $3 \%$ & $55 \%$ & $3 \%$ & $0 \%$ \\
\hline Agencia de Políticas de Calidad & 5 & $7 \%$ & $86 \%$ & $4 \%$ & $0 \%$ & $4 \%$ & $0 \%$ \\
\hline INNOVADOC (Documentos de Innovación Docente) & 6 & $67 \%$ & $7 \%$ & $7 \%$ & $4 \%$ & $15 \%$ & $0 \%$ \\
\hline Datos de Investigación & 7 & $0 \%$ & $13 \%$ & $0 \%$ & $0 \%$ & $83 \%$ & $3 \%$ \\
\hline $\begin{array}{l}\text { Centro de Recursos para el Aprendizaje e Investigación (CRAI- } \\
\text { UB) }\end{array}$ & 8 & $20 \%$ & $33 \%$ & $20 \%$ & $3 \%$ & $20 \%$ & $3 \%$ \\
\hline Planes Docentes - Guías del estudiante & 9 & $73 \%$ & $20 \%$ & $0 \%$ & $7 \%$ & $0 \%$ & $0 \%$ \\
\hline Histórico UB (Anuarios, Memorias...) & 10 & $0 \%$ & $76 \%$ & $14 \%$ & $7 \%$ & $0 \%$ & $3 \%$ \\
\hline Imagen Corporativa y Márquetin UB & 11 & $0 \%$ & $87 \%$ & $10 \%$ & $3 \%$ & $0 \%$ & $0 \%$ \\
\hline
\end{tabular}




\begin{tabular}{|l|c|c|c|c|c|c|c|}
\hline Mediateca - Investigación & 12 & $0 \%$ & $3 \%$ & $60 \%$ & $0 \%$ & $37 \%$ & $0 \%$ \\
\hline Mediateca - Institucional & 13 & $3 \%$ & $17 \%$ & $77 \%$ & $0 \%$ & $3 \%$ & $0 \%$ \\
\hline Cursos de verano de la Universidad de Barcelona & 14 & $60 \%$ & $30 \%$ & $0 \%$ & $7 \%$ & $3 \%$ & $0 \%$ \\
\hline Software de Investigación & 15 & $3 \%$ & $3 \%$ & $3 \%$ & $63 \%$ & $27 \%$ & $0 \%$ \\
\hline $\begin{array}{l}\text { Publicaciones de proyectos de investigación financiados por la } \\
\text { UE }\end{array}$ & 16 & $0 \%$ & $3 \%$ & $17 \%$ & $0 \%$ & $80 \%$ & $0 \%$ \\
\hline Artículos publicados en revistas & 17 & $3 \%$ & $0 \%$ & $30 \%$ & $0 \%$ & $67 \%$ & $0 \%$ \\
\hline Libros / Capítulos de libros & 18 & $10 \%$ & $0 \%$ & $48 \%$ & $0 \%$ & $38 \%$ & $3 \%$ \\
\hline Comunicaciones a congresos & 19 & $3 \%$ & $17 \%$ & $13 \%$ & $7 \%$ & $60 \%$ & $0 \%$ \\
\hline Exposiciones & 20 & $7 \%$ & $48 \%$ & $30 \%$ & $7 \%$ & $7 \%$ & $0 \%$ \\
\hline Documentos de trabajo & 21 & $28 \%$ & $21 \%$ & $14 \%$ & $3 \%$ & $28 \%$ & $7 \%$ \\
\hline Divulgación y Prensa & 22 & $0 \%$ & $52 \%$ & $31 \%$ & $3 \%$ & $14 \%$ & $0 \%$ \\
\hline Software creado por el alumnado & 23 & $14 \%$ & $0 \%$ & $0 \%$ & $72 \%$ & $10 \%$ & $3 \%$ \\
\hline Centro de Estudios Internacionales de la UB & 24 & $23 \%$ & $63 \%$ & $0 \%$ & $0 \%$ & $13 \%$ & $0 \%$ \\
\hline Seminarios de Investigación & 25 & $14 \%$ & $3 \%$ & $3 \%$ & $0 \%$ & $76 \%$ & $3 \%$ \\
\hline Ciencia Histórica de la UB & 26 & $14 \%$ & $52 \%$ & $17 \%$ & $3 \%$ & $10 \%$ & $3 \%$ \\
\hline Tesis Doctorales - Departamento Ecología & 27 & $7 \%$ & $0 \%$ & $0 \%$ & $0 \%$ & $20 \%$ & $73 \%$ \\
\hline Tesis Doctorales - Departamento - Dibujo & 28 & $10 \%$ & $0 \%$ & $0 \%$ & $0 \%$ & $20 \%$ & $70 \%$ \\
\hline Tesis Doctorales - Escuela Universitaria de Enfermería & 29 & $7 \%$ & $0 \%$ & $0 \%$ & $0 \%$ & $20 \%$ & $73 \%$ \\
\hline OMADO (Objetos y Materiales Docentes) & 30 & $83 \%$ & $0 \%$ & $17 \%$ & $0 \%$ & $0 \%$ & $0 \%$ \\
\hline
\end{tabular}

Tabla XII. Matriz de popularidad. Fuente: Elaboración propia.

Se puede apreciar una distribución poco uniforme en la que algunas tarjetas tienen índices de coincidencia relativamente altos con valores de más del $70 \%$, mientras que otras no alcanzan el $30 \%$. Este dato es un primer indicativo de la mala adecuación de la arquitectura del portal a las necesidades de nuestros usuarios ya que denota una notable discordancia en cómo los usuarios pueden reflejar su modelo mental en las categorías que existen actualmente. 


\section{Dendrograma Comunidades y Colecciones}

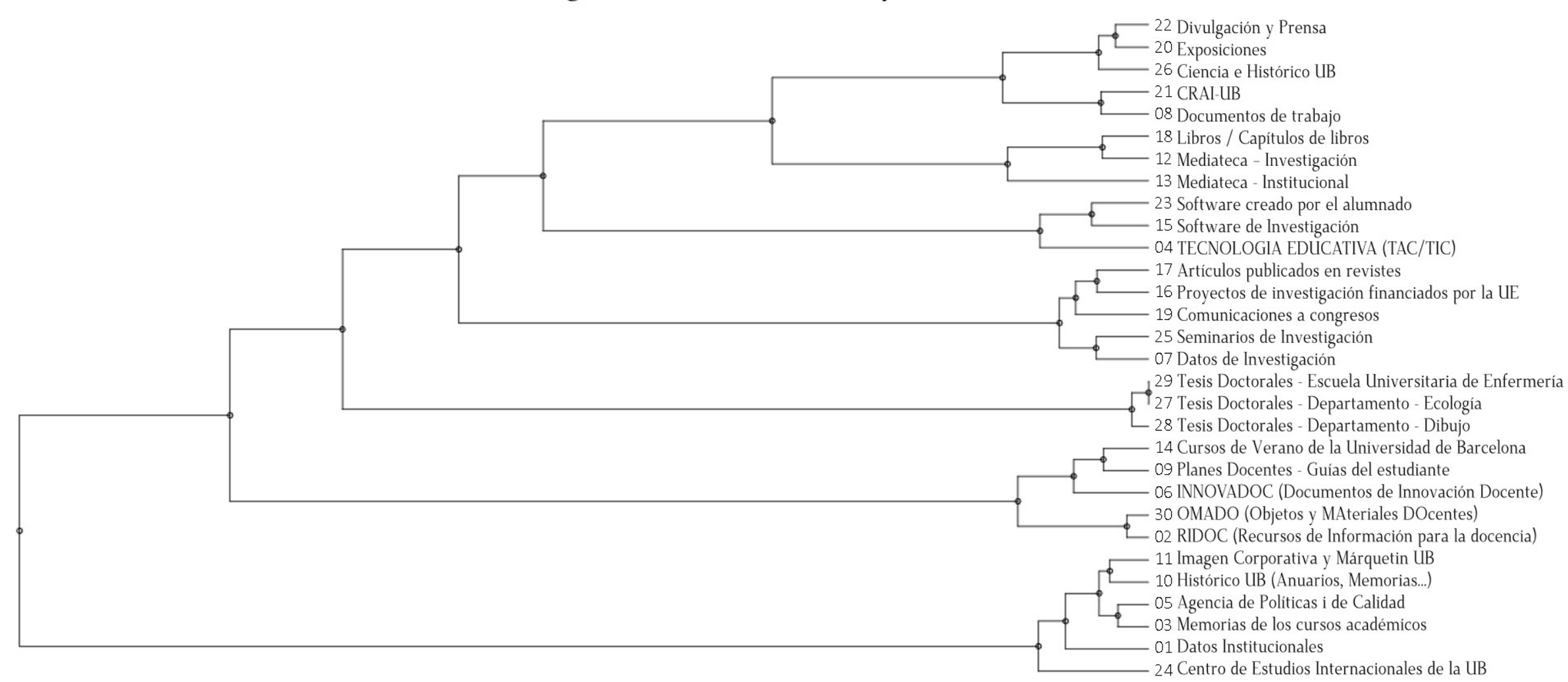


En la tabla XIII se muestran los datos finales obtenidos de cruzar la matriz de popularidad con los clústeres generados en el dendrograma. En ella se aprecian las agrupaciones creadas por los usuarios para cada tarjeta, y las categorías correspondientes según el índice de popularidad.

\begin{tabular}{|c|c|c|c|c|c|c|c|c|c|}
\hline Agrupación & Tarjeta & \multicolumn{8}{|c|}{ Categoría y \% de popularidad } \\
\hline \multirow{3}{*}{1} & Libros / Capítulos de libros & $48 \%$ & \multirow{3}{*}{\multicolumn{3}{|c|}{ Mediateca }} & $38 \%$ & \multirow{3}{*}{\multicolumn{3}{|c|}{ Recerca }} \\
\hline & Mediateca - Investigación & $60 \%$ & & & & $37 \%$ & & & \\
\hline & Mediateca - Institucional & $77 \%$ & & & & $3 \%$ & & & \\
\hline \multirow{3}{*}{2} & Software creado por el alumnado & $72 \%$ & \multirow{3}{*}{\multicolumn{3}{|c|}{ Software }} & $14 \%$ & \multirow{3}{*}{\multicolumn{3}{|c|}{ Docencia }} \\
\hline & Software de Investigación & $63 \%$ & & & & $3 \%$ & & & \\
\hline & TECNOLOGIA EDUCATIVA (TAC/TIC) & $55 \%$ & & & & $38 \%$ & & & \\
\hline \multirow{5}{*}{3} & Artículos publicados en revistes & $67 \%$ & \multirow{5}{*}{\multicolumn{7}{|c|}{ Investigación }} \\
\hline & Proyectos de investigación financiados por la UE & $80 \%$ & & & & & & & \\
\hline & Comunicaciones a congresos & $60 \%$ & & & & & & & \\
\hline & Seminarios de Investigación & $76 \%$ & & & & & & & \\
\hline & Datos de Investigación & $83 \%$ & & & & & & & \\
\hline \multirow{3}{*}{4} & Tesis Doctorales - Escuela Universitaria de Enfermería & $73 \%$ & \multirow{3}{*}{\multicolumn{3}{|c|}{ Tesis }} & & & & \\
\hline & Tesis Doctorales - Departamento - Ecología & $70 \%$ & & & & & & & \\
\hline & Tesis Doctorales - Departamento - Dibujo & $73 \%$ & & & & & & & \\
\hline \multirow{5}{*}{5} & Cursos de Verano de la Universidad de Barcelona & $60 \%$ & \multirow{5}{*}{\multicolumn{3}{|c|}{ Docencia }} & & & & \\
\hline & Planes Docentes - Guías del estudiante & $73 \%$ & & & & & & & \\
\hline & INNOVADOC (Documentos de Innovación Docente) & $67 \%$ & & & & & & & \\
\hline & OMADO (Objetos y Materiales Docentes) & $83 \%$ & & & & & & & \\
\hline & RIDOC (Recursos de Información para la docencia) & $83 \%$ & & & & & & & \\
\hline \multirow{6}{*}{6} & Imagen Corporativa y Márquetin UB & $87 \%$ & \multirow{6}{*}{\multicolumn{3}{|c|}{ Institucional }} & & & & \\
\hline & Histórico UB (Anuarios, Memorias...) & $76 \%$ & & & & & & & \\
\hline & Agencia de Políticas i de Calidad & $86 \%$ & & & & & & & \\
\hline & Memorias de los cursos académicos & $73 \%$ & & & & & & & \\
\hline & Datos Institucionales & $100 \%$ & & & & & & & \\
\hline & Centro de Estudios Internacionales de la UB & $63 \%$ & & & & & & & \\
\hline \multirow{5}{*}{7} & Divulgación y Prensa & $52 \%$ & \multirow{5}{*}{ 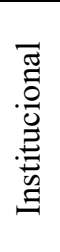 } & $31 \%$ & & $0 \%$ & \multirow{5}{*}{$\begin{array}{l}\cdot \frac{\pi}{0} \\
\stackrel{\Xi}{0} \\
0 \\
0\end{array}$} & $14 \%$ & \multirow{5}{*}{ 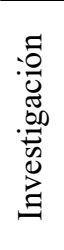 } \\
\hline & Exposiciones & $48 \%$ & & $30 \%$ & ల్ల & $7 \%$ & & $7 \%$ & \\
\hline & Ciencia e Histórico UB & $52 \%$ & & $17 \%$ & . & $14 \%$ & & $10 \%$ & \\
\hline & CRAI-UB & $33 \%$ & & $20 \%$ & $\sum^{0}$ & $20 \%$ & & $20 \%$ & \\
\hline & Documentos de trabajo & $21 \%$ & & $14 \%$ & & $28 \%$ & & $28 \%$ & \\
\hline
\end{tabular}

Tabla XIII. Agrupación de clústeres y porcentajes de popularidad. Fuente: Elaboración propia.

En algunos casos, como las categorías 2 a 6, la asignación de las tarjetas por parte de los usuarios es coincidente, es decir la mayoría de los usuarios han tendido a hacer agrupaciones de tarjetas bajo una misma categoría. Mientras que, para las agrupaciones, 1 y 7 aparecen diferencias notables, y los usuarios han repartido las mismas tarjetas entre distintas categorías creando agrupaciones poco homogéneas.

Esto significa que, en el modelo mental de nuestros usuarios, estas tarjetas deberían estar agrupadas, pero de las categorías existentes en la propuesta actual del repositorio, ninguna resulta adecuada. Si a partir del card sorting de evaluación, se elabora un card sorting para hacer una propuesta nueva de arquitectura es necesario tener en cuenta estos datos ya que cobrará una notable importancia utilizar la metodología de card sorting abierto. De este modo los usuarios podrán proponer etiquetas nuevas para las categorías que les permitan reflejar de mejor manera su modelo mental. 
En la tabla XIV se aprecia la diferencia entre la estructura actual del Depósito Digital y aquella que han propuesto los usuarios. Encontramos diferencias en el 36,66\% (11/30) de las etiquetas analizadas, lo que indica que se puede producir una mala experiencia de usuario en una de cada tres $(1 / 3)$ interacciones.

\begin{tabular}{|c|c|c|c|}
\hline Tarjeta & Categoría Propuesta & Coinciden & Categoría Actual \\
\hline Libros / Capítulos de libros & Mediateca & $\leftrightarrow$ & Investigación \\
\hline Mediateca - Investigación & Mediateca & $\leftrightarrow$ & Mediateca \\
\hline Mediateca - Institucional & Mediateca & $\leftrightarrow$ & Mediateca \\
\hline Software creado por el alumnado & Software & $\leftrightarrow$ & Software \\
\hline Software de Investigación & Software & $\leftrightarrow$ & Software \\
\hline TECNOLOGIA EDUCATIVA (TAC/TIC) & Software & $\leftrightarrow$ & Investigación \\
\hline Artículos publicados en revistes & Investigación & $\leftrightarrow$ & Investigación \\
\hline Proyectos de investigación financiados por la UE & Investigación & $\leftrightarrow$ & Investigación \\
\hline Comunicaciones a congresos & Investigación & $\leftrightarrow$ & Investigación \\
\hline Seminarios de Investigación & Investigación & $\leftrightarrow$ & Institucional \\
\hline Datos de Investigación & Investigación & $\leftrightarrow$ & Datos \\
\hline Tesis Doctorales - Escuela Universitaria de Enfermería & Tesis & $\leftrightarrow$ & Tesis \\
\hline Tesis Doctorales - Departamento - Ecología & Tesis & $\leftrightarrow$ & Tesis \\
\hline Tesis Doctorales - Departamento - Dibujo & Tesis & $\leftrightarrow$ & Tesis \\
\hline Cursos de Verano de la Universidad de Barcelona & Docencia & $\leftrightarrow$ & Institucional \\
\hline Planes Docentes - Guías del estudiante & Docencia & $\leftrightarrow$ & Institucional \\
\hline INNOVADOC (Documentos de Innovación Docente) & Docencia & $\leftrightarrow$ & Docencia \\
\hline OMADO (Objetos y Materiales Docentes) & Docencia & $\leftrightarrow$ & Docencia \\
\hline RIDOC (Recursos de Información para la docencia) & Docencia & $\leftrightarrow$ & Docencia \\
\hline Imagen Corporativa y Márquetin UB & Institucional & $\leftrightarrow$ & Institucional \\
\hline Histórico UB (Anuarios, Memorias...) & Institucional & $\leftrightarrow$ & Institucional \\
\hline Agencia de Políticas i de Calidad & Institucional & $\leftrightarrow$ & Institucional \\
\hline Memorias de los cursos académicos & Institucional & $\leftrightarrow$ & Institucional \\
\hline Datos Institucionales & Institucional & $\leftrightarrow$ & Institucional \\
\hline Centro de Estudios Internacionales de la UB & Institucional & $\leftrightarrow$ & Institucional \\
\hline Divulgación y Prensa & Mediateca & $\leftrightarrow$ & Investigación \\
\hline Exposiciones & Mediateca & $\leftrightarrow$ & Institucional \\
\hline Ciencia e Histórico UB & Mediateca & $\leftrightarrow$ & Institucional \\
\hline CRAI-UB & Mediateca & $\leftrightarrow$ & Investigación \\
\hline Documentos de trabajo & Mediateca & $\leftrightarrow$ & Investigación \\
\hline
\end{tabular}

Tabla XIV. Relación entre arquitectura actual y la propuesta por los usuarios. Fuente: Elaboración propia.

Consideramos por tanto que queda manifiesta la no adecuación del sistema de navegación (y por tanto de una parte del sistema de organización) del repositorio a las necesidades de información específicas de sus usuarios, dificultando la recuperación y generando una mala experiencia de usuario. Todo ello puede tener a medio plazo un impacto negativo en la visibilidad de la producción documental y científica de la institución. 


\section{DISCUSIÓN Y CONCLUSIONES}

Este artículo plantea la utilización de metodologías propias de la disciplina de la arquitectura de la información para mejorar el acceso a los contenidos de los repositorios institucionales. En concreto se propone un método basado fundamentalmente en las técnicas de user persona y card sorting pensado para ser aplicado por cualquier institución que disponga de un repositorio de publicaciones académicas.

A partir de lo expuesto, se pueden extraer una serie de conclusiones y de lecciones aprendidas.

Por un lado, la complejidad de la metodología propuesta puede compensar los costes económicos que derivarían de realizar un card sorting con una herramienta de pago.

Por otro, la metodología propuesta se ha aplicado satisfactoriamente en un estudio de caso y no se han encontrado impedimentos en la gestión y tratamiento de los datos obtenidos.

En tercer lugar, en el estudio de caso ha quedado reflejada la inconsistencia de los modelos organizativos derivados de la implementación directa, sin ningún tipo de elaboración, del software utilizado para la creación del repositorio y su poca adecuación a las necesidades específicas de los usuarios. En el caso del Depósito Digital de la UB, llegando a generar una mala experiencia de usuario en una de cada tres interacciones.

Y, por último, en vista de la disparidad de los resultados obtenidos en algunas agrupaciones hechas por los usuarios, se confirma que para llevar a cabo una propuesta nueva de arquitectura es necesario realizar un card sorting de tipo abierto. De este modo se persigue que los usuarios no se vean obligados a adaptar su modelo mental a una estructura creada previamente.

La propuesta realizada y los resultados obtenidos representan una primera aproximación al uso de la AI en la problemática del acceso a los contenidos de los repositorios institucionales. En este sentido, y con todas las limitaciones propias de un primer estudio exploratorio, este trabajo aporta una perspectiva apenas considerada en la literatura especializada sobre repositorios. Creemos que esta perspectiva debe ser al menos considerada por los gestores de los mismos en aras a una mejora de sus prestaciones, y a contribuir a, en palabras de Delgado LópezCózar, "evitar que los repositorios sean cementerios de documentos, como hasta ahora han sido, salvo honrosas excepciones" (Delgado López-Cozar, 2018).

\section{Agradecimientos}

Este trabajo se ha desarrollado gracias al proyecto "El acceso abierto a la ciencia en España: evaluación de su impacto en el sistema de comunicación científica”, financiado por el Plan Nacional de I+D+i (2015-2018; CSO201452830-P) del Ministerio de Economía y Competitividad del Gobierno de España.

\section{BIBLIOGRAFÍA}

ALJOHANI, M. y BLUSTEIN, J. Personas Help Understand Users' Needs, Goals and Desires in an Online Institutional Repository. World Academy of Science, Engineering and Technology International Journal of Computer and Information Engineering, 2015, vol. 9, $\mathrm{n}^{\mathrm{o}}$ 2, p. 629-636 [en línea]. Disponible en: $<$ https://waset.org/publications/10002376/> [Consulta: 8 de diciembre de 2018].

BARRUECO CRUZ, J.M. et al. Guía para la evaluación de repositorios institucionales de investigación. Madrid: Fecyt, Recolecta, Crue, 2010 [en línea]. Disponible $<$ http://recolecta.fecyt.es/sites/default/files/contenido/documentos/GuiaEvaluacionRecolectav1.0-1.pdf $>$ [Consulta: 8 de diciembre de 2018].

BARRUECO Cruz, J.M. et al. Guía para la evaluación de repositorios institucionales de investigación. Madrid: Fecyt, Recolecta, Crue, 2014 [en línea]. Disponible en: <http://recolecta.fecyt.es/guias $>$ [Consulta: 8 de diciembre de 2018].

BATES, M.J. The design of browsing and berrypicking techniques for the online search interface. Online Review Journal of Documentation Journal of Knowledge Management, 1989, vol. 13, $\mathrm{n}^{\mathrm{o}}$ 2, p. 407-424 [en línea]. Disponible en: https://doi.org/10.1108/eb024320.

BURFORD, S. Complexity and the practice of web information architecture. Journal of the american society for information science and technology, 2011, vol. 62, $\mathrm{n}^{\mathrm{o}} 10, \mathrm{p}$. 2024-2037 [en línea]. Disponible en: $<$ http://onlinelibrary.wiley.com/> [Consulta: 8 de diciembre de 2018]. 
CASSELLA, M. y MORANDO, M. Fostering new roles for librarians: skills set for repository managers — results of a survey in Italy. LIBER Quarterly, 2012, vol. 21, n ${ }^{\mathrm{o}} 3 / 4$, p. 407-428 [en línea]. Disponible en: $<$ http://liber.library.uu.nl/index.php/lq/article/view/8033/8397> [Consulta: 8 de diciembre de 2018].

CORBETTA, P. Metodología y técnicas de investigación social. $2^{\mathrm{a}}$ Edición. Madrid: McGraw-Hill, 2007.

DELGADO LÓPEZ-CÓZAR, E. La hoja de ruta verde de la comunicación científica ¿qué podemos hacer autores, bibliotecarios, gestores y agencias de evaluación para cambiar las cosas? Aula Magna 2.0, 2018 [en línea]. Disponible en: $<$ https://cuedespyd.hypotheses.org/5397> [Consulta: 8 de diciembre de 2018].

EXPÓSITO-GARCÍA, A. y VELASCO-MORENTE, F. How efficient are universities at publishing research? A data envelopment analysis of Spanish state universities. El profesional de la información, 2018, vol. 27, n $\mathrm{n}^{\circ}$ 5, p. 11081115 [en línea]. Disponible en: https://doi.org/10.3145/epi.2018.sep.14.

FARKAS, K.D. y FARKAS, B.J. Guidelines for designing web navigation. Technical communication, 2000, vol. 47, $\mathrm{n}^{\mathrm{o}} 3$, p. 341-358.

GARCÍA, R.; BOTELLA, F. y MARCOS, M.-C. Hacia la arquitectura de la información 3.0: pasado, presente y futuro. El profesional de la información, 2010, vol. 19, $\mathrm{n}^{\mathrm{o}} 4$, p. 339-347 [en línea]. Disponible en: $<$ http://www.elprofesionaldelainformacion.com/contenidos/2010/julio/02.html $>$ [Consulta: 8 de diciembre de 2018].

GARRETT, J.J. The Elements of User Experience: User-Centered Design for the Web and Beyond. ( $2^{\mathrm{a}}$ edición). Berkeley, CA: New Riders, 2011, [en línea]. Disponible en: https://doi.org/10.1145/889692.889709.

GUALLAR, J.; FERRAN-FERRER, N.; ABADAL, E. y SERVER, A. Revistas científicas españolas de información y documentación: análisis temático y metodológico. El profesional de la información, 2017, vol. 26, nº 5, p. $947-960$ [en línea]. Disponible en: https://doi.org/10.3145/epi.2017.sep.16.

HASSAN MONTERO, Y.; MARTÍN FERNÁNDEZ, F.J. y MARTÍN RODRÍGUEZ, Ó. Clasificaciones facetadas y metadatos (I): Conceptos básicos. No solo usabilidad, 2003 [en línea]. Disponible en: $<$ http://www.nosolousabilidad.com/articulos/clas_facetadas1.htm > [Consulta: 8 de diciembre de 2018].

JULIEN, H. Students' information needs and behavior. Encyclopedia of Library and Information Sciences $\left(3^{\mathrm{a}}\right.$ edición), 2009, p. 5059-5064 [en línea]. Disponible en: https://doi.org/10.1081/E-ELIS3-120043657.

KIM, Y.H. y KIM, H.H. Development and validation of evaluation indicators for a consortium of institutional repositories: a case study of collection. Journal of the American Society for Information Science \& Technology, 2008, vol. 59, no 8, p. 1282-1294 [en línea]. Disponible en: http://dx.doi.org/10.1002/asi.20818.

KRIKELAS, J. Information seeking behavior: patterns and concepts. Drexel library quarterly, 1983, vol. 19, p. 5-20.

LUCA, E. y NARAYAN, B. Redesigning the open-access institutional repository: A user experience approach. Digital Libraries: Knowledge, Information, and Data in an Open Access Society, 2016, p. 275-281 [en línea]. Disponible en: https://doi.org/10.1007/978-3-319-49304-6_33.

MILLER, C.S. y REMINGTON, R.W. Modeling information navigation: implications for information architecture. Human-computerinteraction, 2004, vol. 19, $\mathrm{n}^{\mathrm{o}} \quad 3, \quad$ p. 225-271 [en línea]. Disponible en: http://doi.org/10.1207/s15327051hci1903_2.

MORVILLE, P. y ROSENFELD, L. Information architecture for the world wide web (3ª edición). Estados Unidos: O'Reilly Media Inc., 2007.

NIELSEN, J. IA Task failures remain costly. Nielsen Norman Group, 2009 [en línea]. Disponible en: $<$ https://www.nngroup.com/articles/ia-task-failures-remain-costly/> [Consulta: 8 de diciembre de 2018].

NIELSEN, J. How many test users in a usability study. Nielsen Norman Group, 2012 [en línea]. Disponible en: $<$ http://www.useit.com/alertbox/number-of-test-users.html $>$ [Consulta: 8 de diciembre de 2018].

PÉREZ-MONTORO, M. Arquitectura de la información en entornos web. Gijón: Trea, 2010.

PÉREZ-MONTORO, M. y CODINA Ll. Navigation design and SEO for content-intensive websites: A guide for an efficient digital communication. Chandos Publishing, 2016.

PINFIELD, S. et al. Open-access repositories worldwide, 2005-2012: Past growth, current characteristics, and future possibilities. Journal of the Association for Information Science and Technology, 2014, vol. 65, $\mathrm{n}^{\circ}$ 12, p. 24042421 [en línea]. Disponible en: https://doi.org/10.1002/asi.23131.

PRIMARY RESEARCH GROUP. The survey of institutional digital repositories, 2012-13 Edition. New York: Primary Research Group, 2012.

ROVIRA, C. y MARCOS, M.-C. Diseño de sitios web: disciplinas, materias y esquemas integradores. Hipertext.net, 2013, $\mathrm{n}^{\mathrm{o}} 11$ [en línea]. Disponible en: <https://www.upf.edu/hipertextnet/numero-11/Diseno_sitios_web.html> [Consulta: 8 de diciembre de 2018].

ROSENFELD, L.; MORVILLE, P. y ARANGO, J. Information architecture: For the web and beyond. Sebastopol (4a edición). California, Estados Unidos: O’Reilly Media, 2015.

RUSSELL-ROSE, T. Designing search: Results pages. UX Magazine, 2013, nº 1124. ISSN: $2168-5681$ [en línea]. Disponible en: $<$ https://uxmag.com/articles/designing-search-results-pages> [Consulta: 8 de diciembre de 2018].

RUSSELL-ROSE, T. y TYLER, T. Designing the search experience: The information architecture of discovery $\left(1^{\mathrm{a}}\right.$ edición). Burlington, Estados Unidos: Morgan Kaufmann, 2013. 
SERRANO-VICENTE, R.; MELERO, R. y ABADAL, E. Indicadores para la evaluación de repositorios institucionales de acceso abierto. Anales de Documentación, 2014, vol. 17, no 2, p. 1-12 [en línea]. Disponible en: https://doi.org/10.6018/analesdoc.17.2.190821.

SERRANO-VICENTE, R.; MELERO, R. y ABADAL, E. Evaluation of Spanish institutional repositories based on criteria related to technology, procedures, content, marketing and personnel. Data Technologies and Applications, 2018, vol. 52, no 3, p. 384-404 [en línea]. Disponible en: https://doi.org/10.1108/DTA-10-2017-0074.

SPENCER, D. card sorting. Designing usable categories. New York: Rosenfeld Media, 2009.

TORRES-SALINAS, D.; GARCÍA-MORENO, J.; ROBINSON-GARCÍA, N.; DELGADO-LÓPEZ-CÓZAR, E. y HERRERA, F. Rankings ISI de las universidades españolas según campos y disciplinas científicas. El profesional de la informacion, 2011, vol. 20, $\mathrm{n}^{\mathrm{o}}$ 6, p. 701-711 [en línea]. Disponible en: https://doi.org/10.3145/epi.2011.nov.16.

TRAMULLAS, J. Documentos y servicios digitales: de la usabilidad al diseño centrado en el usuario. El profesional de la información, 2003, vol. 12, $\mathrm{n}^{\mathrm{o}} 2$ (marzo-abril), p. 107-110 [en línea]. Disponible en: $<$ http://www.elprofesionaldelainformacion.com/contenidos/2003/marzo/3.pdf $>$ [Consulta: 8 de diciembre de 2018].

TULLIS, T. y WOOD, L. How many users are enough for a card-sorting study? Usability Professionals Association (UPA) 2004-Conference, Minneapolis, 2004, junio, vol. 7-11.

WESSA, P. Free Statistics Software, Office for Research Development and Education, versión 1.2.1. 2018 [en línea]. Disponible en: <https://www.wessa.net/> [Consulta: 8 de diciembre de 2018]. 